\title{
Music Suggestion Via Sentimental Analysis of User-Inputted Texts
} doi : https://doi.org/10.32628/CSEIT217320

\author{
Vipin Wani*, Niketan Bothe, Avani Soni \\ SOCSE, Sandip University, Nashik, Maharashtra, India
}

\section{Article Info}

Volume 7, Issue 3

Page Number: 51-66

\section{Publication Issue :}

May-June-2021

\section{Article History}

Accepted : 01 May 2021

Published : 07 May 2021

\section{ABSTRACT}

This paper overviews the state of craftsmanship in feeling acknowledgment from content and give music. Music is oftentimes alluded to as a "language of emotion", and it is characteristic for us to classify music in terms of its enthusiastic affiliations. This paper, investigations the utilize of Natural Language Processing (NLP) for dismember the human dialect and make information models out of it. But to develop a computer program which is able give music based on text's feeling. There may be impressive difference with respect to the recognition and translation of the feelings of a melody or uncertainty inside the piece itself. In this paper we provide a platform that tailors music according to a user-specific emotion, while also opening up the user to music they might not have perceived earlier on in life - the powers of recommendation and discovery in one piece of technology.

Keywords : Music recommendation, emotion analysist, Sentiment analysis

\section{INTRODUCTION}

Music and automated machine learning have been a domain combination that has, for the better part of the last decade or so, not been explored to its fullest. The addition of data analysis to this makes the entire domain an overlooked entity that merits more depth in research and application. In retrospect,

machine learning/deep learning algorithms have always been used to implement Natural Language Processing (NLP), which is used to dissect the human language and create data models out of it, for greater uses. As mentioned, data analysis works hand-inhand with Machine Learning. The human psyche is a vast spectrum - the ability to emote is what makes us human. Music is globally considered to be a valve for emotional release, and thus it only seems fair that the amalgam of music, technology and data analysis should create something riveting for the common human being.

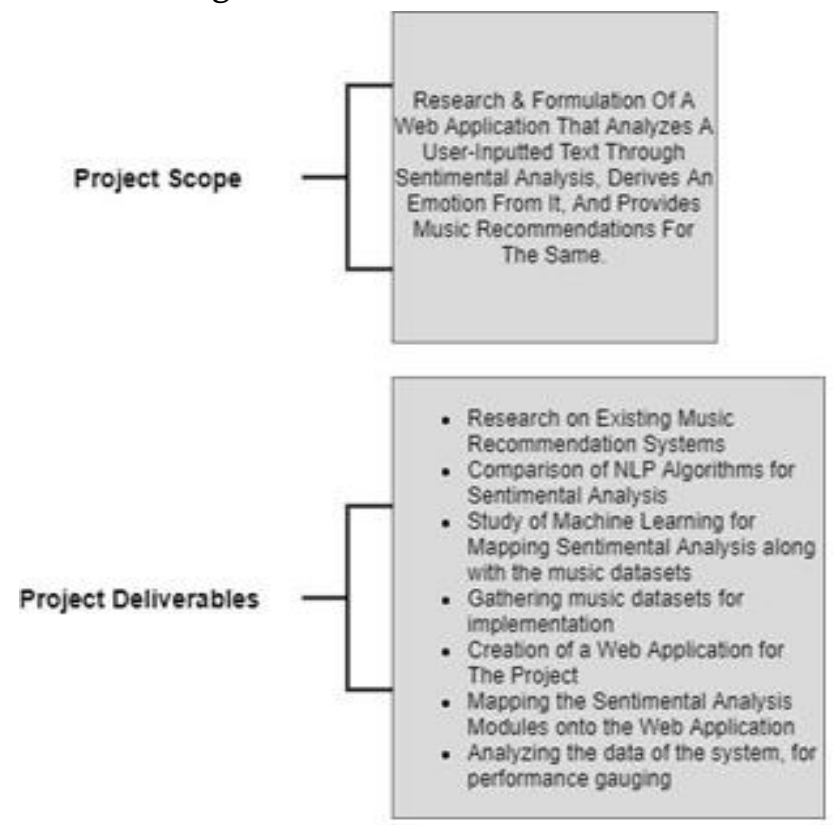

The fusion of arts with technology has not only been a hot topic of debate, but an avenue that has been 
slowly simmering under its top for a very long time now. Music production has, to a large extent, used technology to its fullest, or even more so, is still to see the real power of technology harnessing the best out of it. Along similar lines, applications that fuse music and technology into one as an everyday element for the masses have captured the world - be it Apple, Spotify or any other such application which provides music to the people of the world at nominal prices. Music recommendation falls onto that same plate - there lies potential untapped when you consider the spectrum of present and future technology. There lies so much beyond simply typing in a keyword or trying to remember what genre a certain song was.

Music and automated machine learning have been a domain combination that has, for the better part of the last decade or so, not been explored to its fullest. The addition of data analysis to this makes the entire domain an overlooked entity that merits more depth in research and application. The human psyche is a vast spectrum - the ability to emote is what makes us human. Music is globally considered to be a valve for emotional release, and thus it only seems fair that the amalgam of music, technology and data analysis should create something riveting for the common human being. Music Recommendation Systems often choose ratings as a mode of analysis, which is often overlooked by users.

\section{LITERATURE REVIEW}

[Paper1] The objective of this paper is to investigate when and what music a user will listen to. We propose a fine-grained time-aware music recommendation model (FTAMR). We improve the recommendation qualities from two sides: 1) User 2) Song

We calculate asymmetric co-recommendation probabilities and propose the $\mathrm{k}$-means clustering based on items of classify songs into different categories. FTAMR (Fine grained Time aware music recommendation), explores the user's habit in a period and then recommends different songs at different times. This improves pure CF some aspects 1) employing asymmetric co-recommendation probability to deal with different reciprocity between two songs. 2) It utilizing k-mean clustering to treat each song different.

[Paper 2] This paper proposes a novel model classification technique using the improved Rocchino algorithm. Addressing the problem by considering the nearest vector along with a prototype vector. This Method validate using real music data/ collected from well know music portals. The model for music classifies the utilities metadata information, lyrics of song \& social tags for determining the mood using rocchio algorithm. The proposed framework for music mood classification using Rocchino algorithm. Utilized the meta data \& lyrics semantic for mood classification. It simulates and compares various classification algorithms. It gives better performance than other approaches. It incorporates audio features such as frequency and pitch would improve.

[Paper 3] It works on classifying on the greatest feature of music the lyrics. It uses about 11,000 lyrics based on the billboard chart from 1 to 100 . The natural language processing work was done to refine each of the lyrics and music was digitized as sentimental and emotion. It selects the new song from the list using the $\mathrm{k}$-nearest neighbors $(\mathrm{K}-\mathrm{NN})$ algorithm. In this approach they consider each piece of music as each genre using text mining. The system has gathered a total of 10,891 lyrics based on Billboard charts and 3,771413 number of words for text mining. It uses stop word NLP and for analyzed the lyrics of music based on lexicon. The K-nearest neighbor (K-NN) algorithm will be used to tell music with the nearest distance. 
[Paper 4] The objective is to filter the explicit content from the music. Propose bagging with selective vocabulary model out performed not only the other competing models designed but also the filtering method that used the man-made profanity dictionary. Bayesian filtering text censoring system carried out the most relevant censoring with least number of errors compared to chi square and list-based censoring system. The lexical Syntactic features (LSF) architecture to detect offensive contents and identify potential offensive users in social media. We designed bugging and adiabats models that automatically determine fail/pass in the lyrics by learning previously labelled data without a predefined pro faintly dictionary. The model vast outperformed the filtering method that used the profanity dictionary. To detect explicit contents in music lyrics more efficiently they applied of score as the value of vectors and use a selective vocabulary set based on postages. The model could distinguish the various explicit content that the filtering method with dictionary cannot capture.

[Paper 5] Objective is to find favorite music from the massive music data on the internet. The proposed algorithm is for personalized music recommendation based on tag information. Uses the improved userbased collaborative filtering algorithm to deal with the user's long-term preferences. Experiments on real dataset demonstrate the efficiency and effectiveness of the improved algorithm. By clustering the user in advance, it can effectively reduce the calculation of the similarity between users, and improve the running speed of the algorithm. It is beneficial to improve the accuracy of the recommendation. Results show that the algorithm proposed in the paper can effectively improve the accuracy and speed of the algorithm. It also improves the quality of music recommendation and user experience.

[Paper 6] The Objective is the result of the recommendation system should be improved and considered in the most of the personalized music recommendation system. The method RTCF (Tagdriven Collaborative filtering recommendation System) analyses the historical records of the tracks of the users in the data sets. The tags have related counts when describing a song or an artist. Different tags can describe the characteristics of the track and artist. The tags not only serve as additional to broaden the content of music but build personalized music recommendations for users. They mined top tags related to the artist and tracks and found that each tag owns a fixed count to reflect the correlation degree of the artist or track.

[Paper 7] The objective of this paper is to investigate Whatever we do, whatever we say, somehow does reflect some of our emotions, using this emotional data for analyzing the Emotions also form an interdisciplinary called Affective Computing. This paper summarizes the previous works done in the field of textual emotion analysis based on various emotional models and computational approaches used. Text emotion analysis also introduces some challenges in our work in the sense that emotions and the ways to express these emotions are all subjective. The emotion analysis uses the natural language processing, text analysis and various computational techniques to determine the emotions hidden in a particular text.

[Paper 8] The objective of this paper is to investigate Whatever we do, whatever we say, somehow does reflect some of our emotions, using this emotional data for analyzing the Emotions also form an interdisciplinary called Affective Computing. This paper summarizes the previous works done in the field of textual emotion analysis based on various emotional models and computational approaches used. Text emotion analysis also introduces some challenges in our work in the sense that emotions and the ways to express these emotions are all subjective. The emotion analysis uses the natural language processing, text analysis and various 
computational techniques to determine the emotions hidden in a particular text. Text emotion analysis also introduces some challenges in our work in the sense that emotions and the ways to express these emotions are all subjective. The emotion analysis uses the natural language processing, text analysis and various computational techniques to determine the emotions hidden in a particular text.

[Paper 9] Human express the emotion in the form of facial expression, speech and writing text. Recently, researchers in computational linguistic (CL) areas are interested in the attention of emotion for Sentiment Analysis (SA). The wide areas of CL research, actually considerable for investigating the emotion dimension detection and searching the approaches and techniques in the term of emotion recognition (ER). The exclusive work on emotion detection is comparatively rare and lacks empirical evaluation research. Analysis, mainly focuses on information retrieval and knowledge discovery from text; 2) Computer Assisted Creativity; 3) Text to Speech generation; and 4) Improvement of HCI. To build emotion dimension dataset, to obtain the optimally of affective computing systems and perform reviews analysis. 1) Keyword spotting techniques, this method has several common the process of keyword marking from the original text to get categories of emotion detection; 2) Lexical affinity method, this way investigates emotion detection based on related keywords.

[Paper 10] In this paper an improved algorithm for personalized music recommendation based on tag information is proposed. We use the improved userbased collaborative filtering algorithm to deal with the user s long- term preferences. Secondly, according to the user- tag-music relationships, getting the music that associated with the user via recommendation algorithm based on bipartite graph. Experiments on real dataset demonstrate the efficiency and effectiveness of the improved algorithm, getting better results and have practical significance. The personalized music recommendation algorithm based on the combination of the user-based collaborative filtering and recommendation algorithm based on bipartite graph can be used to further improve the quality of the music recommendation. K-means clustering algorithm to cluster users, and then use the userbased collaborative filtering algorithm to recommend music.

[Paper 11] The advancement in communication networks and the spread usage of social web is the reason of massive number of emotions that can be extracted frequently. The paper focused on the textual emotion detection as a task of sentiment analysis. It explored the latest state of art approaches for emotion detection in text, and discussed their classification according to the used techniques, the used emotional model and the different used datasets. It a natural-language processing (NLP) task that classify these opinions and emotions into either positive, negative or neural The field of sentiment analysis is composed of primary two subfields; rational sentiment words, and concepts, the opinion mining is related to the rational field and it can be further decomposed into subjectivity detection opinion polarity classification.

[Paper 12] They have tried to fill the gap between emotion recognition and emotion correlation mining through natural language text from Web news. The correlation among emotions, expressed as the confusion and evolution of emotion, is primarily caused by human emotion cognitive bias. The emotion confusion law is extracted through an orthogonal basis. The method is validated using three datasets: 1) the titles; 2) the bodies; and 3) the comments of news articles, covering both objective and subjective texts in varying lengths (long and short). The experimental results show that in subjective comments, emotions are easily mistaken as anger Findings could provide insights for applications regarding affective interaction, such as network 
public sentiment, social media communication, and human-computer interaction.

[Paper 13] The challenging module in CAS (computer-aided services) has recognized the emotion from the signals of speech. In SER (speech emotion recognition), several schemes have used for extracting emotions from the signals, comprising various classification \& speech analysis methods. This manuscript represents an outline of methods \& explores some contemporary literature where the existing models have used for emotion recognition based on speech. Manifold modalities might capture the expressed emotions accurately and result in optimal outcomes of recognition than unimodal methods. Several contributions concentrated on utilizing modalities of audio-visual for recognition of emotion. This could deliberate as a transfer learning strategy, same as word embedding pretraining in NLP (Natural language processing) or pretraining methods on ImageNet for recognition of object.

\section{APPROACH}

\section{A. Naive Bayes Classifiers}

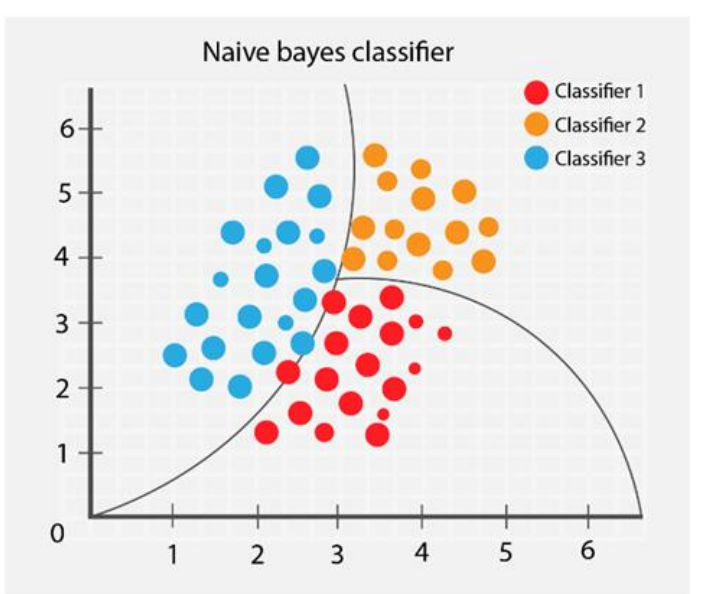

Figure 1. Graphical representation of Naive bayers classifier

The best arrangements are ordinarily the foremost effective ones, and Naïve Bayes may be a great illustration of that. In spite of the progresses in Machine Learning within the final a long time, it has demonstrated too not as it were be straightforward but too quick, exact, and dependable. It has been successfully utilized for numerous purposes, but it works especially well with characteristic dialect handling (NLP) issues. [4] Naïve Bayes could be a probabilistic machine learning calculation based on the Bayes Hypothesis, utilized in a wide assortment of classification errands. In this article, we'll get it the Naïve Bayes calculation and all basic concepts so that there's no room for questions in understanding.[4].

\section{B. Bayes Theorem}

It may be a hypothesis that works on conditional likelihood. Conditional likelihood is the likelihood that something will happen, given that something else has as of now happened. The conditional likelihood can donate us the likelihood of an occasion utilizing its earlier information.[5].

\section{Conditional probability}

1) Were, $P(H)$ : The probability of hypothesis $H$ being true. This is known as prior probability. $\mathrm{P}(\mathrm{E})$ : The probability of the evidence. $\mathrm{P}(\mathrm{E} \mid \mathrm{H})$ : The probability of the evidence given that hypothesis is true. $\mathrm{P}(\mathrm{H} \mid \mathrm{E})$ : The probability of the hypothesis given that the evidence is true.

2) Batch size - total number of training samples in a single backward/forward pass

3) Learning rate - user-defined constant to control the learning rate of the neuron

4) Decay - The ratio between learning rate and epoch. 


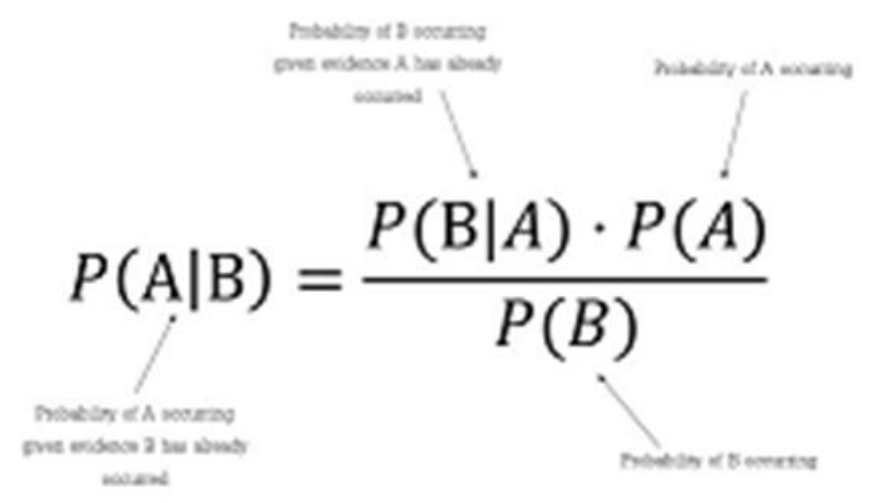

K- Clustering Means

\section{K- Clustering Means}

Clustering is one of the foremost common exploratory data analysis method utilized to induce an instinct approximately the structure of the information. It can be characterized as the errand of identifying subgroups within the information such that data points within the same subgroup (cluster) are exceptionally comparable whereas information focuses totally different clusters are exceptionally distinctive.[5] In other words, we attempt to discover homogeneous subgroups inside the information such that information focuses in each cluster are as comparable as conceivable agreeing to a closeness degree such as Euclidean-based separate or correlation-based remove.[4] The choice of which closeness degree to utilize is application-specific. Clustering investigation can be done on the premise of highlights where we attempt to discover subgroups of tests based on highlights or on the premise of tests where we attempt to discover subgroups of highlights based on tests.[6]

We'll cover here clustering based on highlights. Clustering is utilized in advertise division; where we attempt to discover clients that are comparative to each other whether in terms of behaviors or traits, picture segmentation/compression; where we attempt to gather comparable locales together, record clustering based on themes, etc. Not at all like administered learning, clustering is considered an unsupervised learning strategy since we don't have the ground truth to compare the yield of the clustering calculation to the genuine names to assess its performance.[4] $\mathrm{We}$ as it were need to undertake to explore the structure of the information by gathering the data points into particular subgroups. In this post, we are going cover as it were Kmeans which is considered as one of the foremost utilized clustering calculations due to its simplicity.[5].

Kmeans Algorithm Kmeans calculation is an iterative calculation that tries to segment the dataset into Kpre-defined particular non-overlapping subgroups (clusters) where each information point has a place to as it were one bunch.[3] It tries to create the intracluster information focuses as comparable as conceivable whereas moreover keeping the clusters as distinctive (distant) as conceivable. It relegates information focuses to a cluster such that the entirety of the squared remove between the information focuses and the cluster's centroid (math cruel of all the information focuses that have a place to that cluster) is at the least. The less variety we have inside clusters, the more homogeneous (comparative) the information focuses are inside the same cluster.[6] The way kmeans algorithm works is as follows:

- Specify number of clusters K.

- Initialize centroids by to begin with rearranging the dataset and after that arbitrarily selecting $\mathrm{K}$ information focuses for the centroids without replacement.

- Keep repeating until there's no alter to the centroids. i.e., task of information focuses to clusters isn't changing.

- Compute the entirety of the squared remove between information focuses and all centroids.

- Assign each information point to the closest cluster (centroid).

- Compute the centroids for the clusters by taking the normal of the all-data focuses that have a place to each cluster.[6] 
The objective function is:

$$
J=\sum_{i=1}^{m} \sum_{k=1}^{K} w_{i k}\left\|x^{i}-\mu_{k}\right\|^{2}
$$

where wik=1 for data point $x i$ if it belongs to cluster $\mathrm{k}$; otherwise, wik=0. Also, $\mu \mathrm{k}$ is the centroid of xi's cluster.

It's a minimization problem of two parts. We first minimize J w.r.t. wik and treat $\mu \mathrm{k}$ fixed. Then we minimize J w.r.t. $\mu \mathrm{k}$ and treat wik fixed. Technically speaking, we differentiate J w.r.t. wik first and update cluster assignments (E-step).[5] Then we differentiate J w.r.t. $\mu \mathrm{k}$ and recompute the centroids after the cluster assignments from previous step (M-step). Therefore, E-step is:

In other words, assign the data point xi to the closest cluster judged by its sum of squared distance from cluster's centroid.

And M-step is:

$$
\begin{gathered}
\frac{\partial J}{\partial w_{i k}}=\sum_{i=1}^{m} \sum_{k=1}^{K}\left\|x^{i}-\mu_{k}\right\|^{2} \\
\Rightarrow w_{i k}= \begin{cases}1 & \text { if } k=\operatorname{argmin}_{j}\left\|x^{i}-\mu_{j}\right\|^{2} \\
0 & \text { otherwise. }\end{cases}
\end{gathered}
$$

\section{E. FTAMR (Fine Grained Time Aware Music Recommendation)}

Fine-grained time-aware music suggestion (FTAMR) demonstrate which can extricate the transient music tuning in propensities, both in short-term and in long-term. [5] That's to say, it is able to tell the music sorts to which the client needs to tune in, in a certain time in a day. It makes strides collaborative sifting from two angles: (1) utilizing asymmetric corecommendation likelihood rather than symmetric likeness; (2) clustering melodies instead of looking for $\mathrm{k}$ nearest-neighbors for each song, which helps to address the "less rating" challenge. They offer assistance to realize distant better; a much better; a higher; a stronger; an improved">a distant better proposal. 3) Based on the over works, we conduct broad tests in Last.fm (web site giving millions of melodies) to approve the adequacy of the proposed FTAMR show. [4] It moreover picks up three valuable discoveries that can direct future investigate of finegrained recommendation.

\section{ANALYSIS}

Although a conclusion may review the main points of the paper, do not replicate the abstract as the conclusion. A conclusion might elaborate on the importance of the work or suggest applications and extensions. Authors are strongly encouraged not to call out multiple figures or tables in the conclusion these should be referenced in the body of the paper.

\section{ANALYSIS}

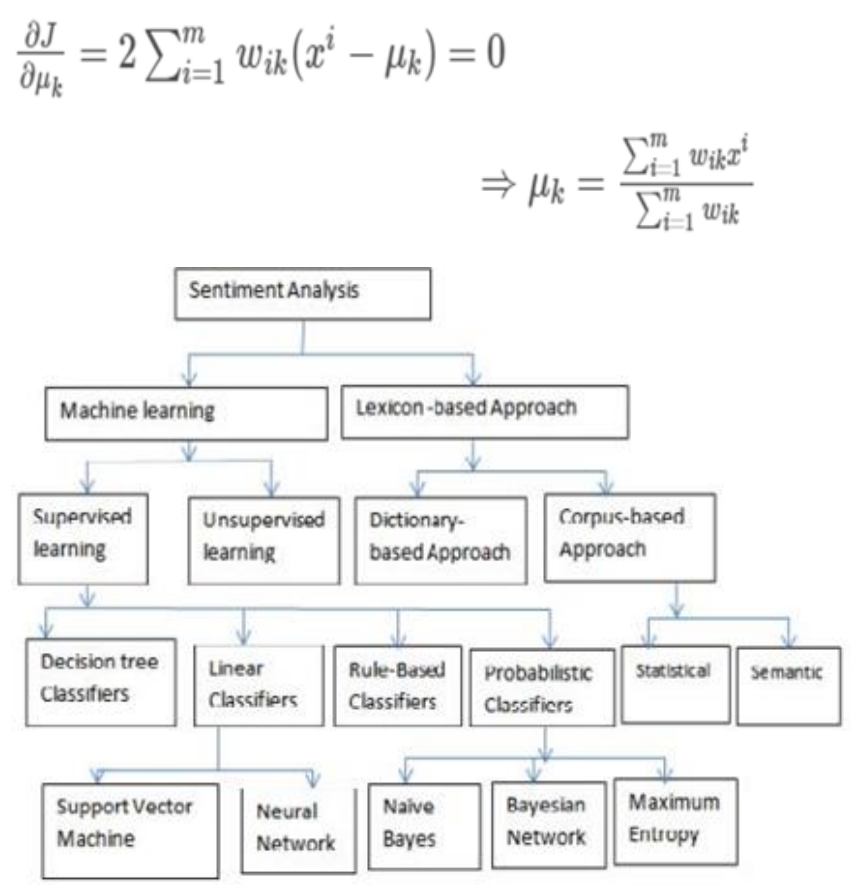

Sentimental analysis can be classified into two typesMachine learning approaches and Lexicon-based approaches. The first step was to introduce a Machine Learning model that did Natural Language Processing, more commonly known as NLP, on the user-inputted text on the web application. The need for Natural 
Language Processing arose when the accuracy of the sentimental analysis was affected by unnecessary words out of context in the sentence. Words that hold only grammatical value, punctuation and other stop words needed to be removed. A Natural Language Processing pipeline was created for this purpose which is illustrated below: Sentence Segmentation -> Word Tokenization -> Lower casing -> Stop words Removal -> Remove Punctuation -> Stemming $\rightarrow$ Lemmatization $\rightarrow$ Parts of Speech Prediction -> Strings to Vectors -> Training Model.

\section{A. SENTENCE SEGMENTATION}

The first step in the pipeline is to break the text apart into separate sentences.

\section{B. WORD TOKENIZATION}

The next step in our pipeline is to break this sentence into separate words or tokens. This is called tokenization.

\section{PREDICTING PARTS OF SPEECH FOR EACH TOKEN}

Next, we'll look at each token and try to guess it's part of speech - whether it is a noun, a verb, an adjective and so on. Knowing the role of each word in the sentence will help us start to figure out what the sentence is talking about.

We can do this by feeding each word (and some extra words around it for context) into a pre-trained partof-speech classification model:

Take a sentence, for example - "London is the capital and most populous city of England and the United Kingdom.”.

London is the capital and most populous city of England and the United Kingdom.

\section{TEXT LEMMATIZATION}

When working with text in a computer, it is helpful to know the base form of each word so that you know that both sentences are talking about the same concept. Otherwise, the strings "pony" and "ponies" look like two totally different words to a computer. In NLP, we call finding this process lemmatization figuring out the most basic form or lemma of each word in the sentence.

\section{E. IDENTIFYING STOP WORDS}

Next, we want to consider the importance of each word in the sentence. English has a lot of filler words that appear very frequently like "and", "the", and "a". When doing statistics on text, these words introduce a lot of noise since they appear way more frequently than other words. Some NLP pipelines will flag them as stop words - that is, words that you might want to filter out before doing any statistical analysis.

Stop words are usually identified by just by checking a hardcoded list of known stop words. But there's no standard list of stop words that is appropriate for all applications. The list of words to ignore can vary depending on your application.

\section{F. DEPENDENCY PARSING}

The next step is to figure out how all the words in our sentence relate to each other. This is called dependency parsing. Dependency parsing is the process of analysing the grammatical structure of a sentence based on the dependencies between the

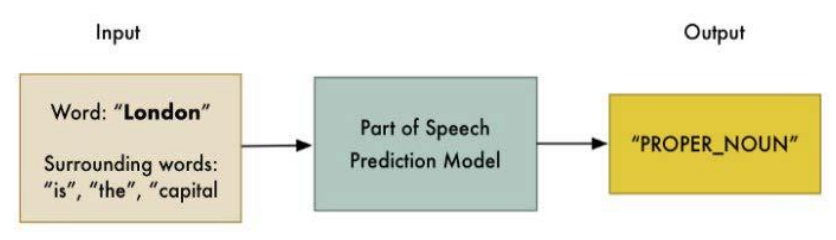

words in a sentence.

\section{G. FINDING NOUN PHRASES}

So far, we've treated every word in our sentence as a separate entity. But sometimes it makes more sense to group together the words that represent a single idea 
or thing. We can use the information from the dependency parse tree to automatically group together words that are all talking about the same thing.

\section{H. NAMED ENTITY RECOGNITION (NER)}

In the example sentence, we have the following nouns:

The goal of Named Entity Recognition, or NER, is to detect and label these nouns with the real-world concepts that they represent.

NER systems use the context of how a word appears in the sentence and a statistical model to guess which type of noun a word represents.

\section{COREFERENCE RESOLUTION}

Coreference resolution is the task of finding all expressions that refer to the same entity in a text. It is an important step for a lot of higher-level NLP tasks that involve natural language understanding such as document summarization, question answering, and information extraction.

\section{J. STRING TO VECTOR}

The outcome of the pipeline was a sentence with only contextual words like the objects in the scene, mood, subjects, scenic description etc. But this was not enough to use it with the model since computers only understand numeric, the need to vectorize the string was rose. This was taken care by a framework called Text Blob.

\section{CLASSIFICATION}

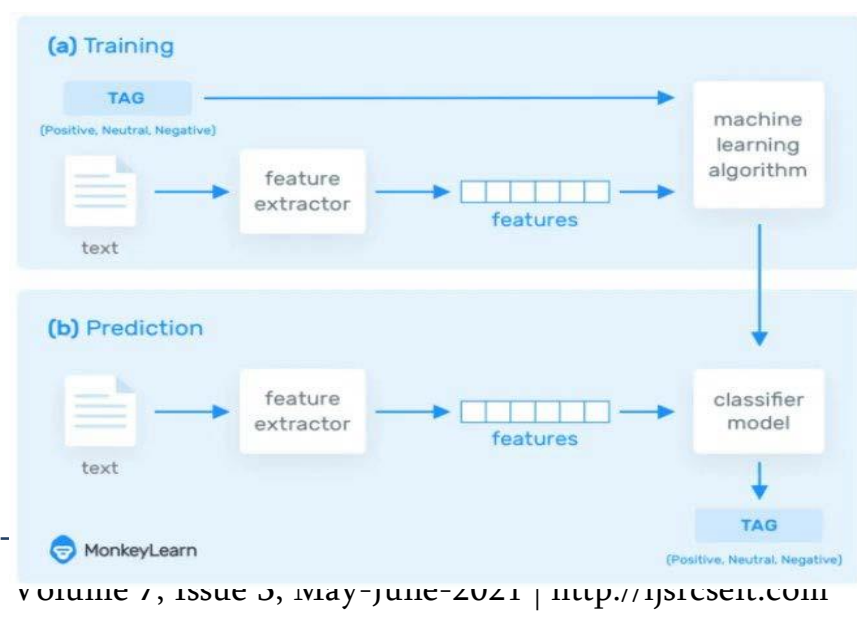

Naive Bayes is a fairly simple group of probabilistic algorithms that, for sentiment analysis classification, assigns a probability that a given word or phrase should be considered positive or negative. Essentially, this is how Bayes' theorem works.

The probability of $A$, if $B$ is true, is equal to the probability of $B$, if $A$ is true, times the probability of A being true, divided by the probability of $\mathrm{B}$ being true

Basically, Naive Bayes calculates words against each other. So, with machine learning models trained for word polarity, we can calculate the likelihood that a word, phrase, or text is positive or negative. When techniques like lemmatization, stop word removal, and TF-IDF are implemented Naive Bayes becomes more and more predictively accurate.

\section{DATA PROCESSING}

For our project's final phase, we used a public Spotify song dataset available on Kaggle, the link for which we have provided below:

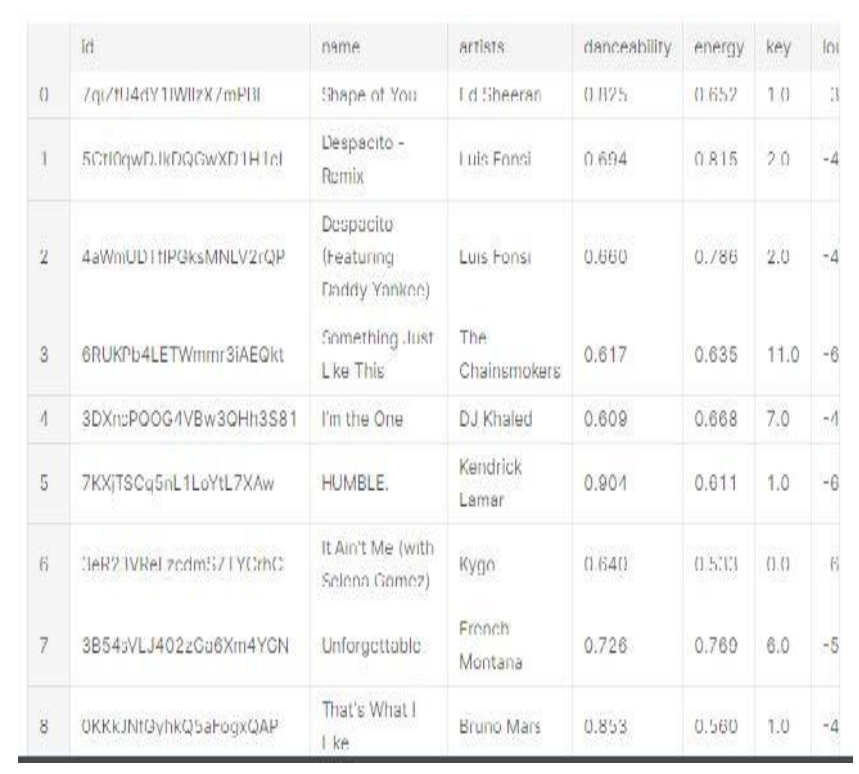

Upon researching, we observed that Spotify ranks and classifies all the songs in its database using a number of audio feature parameters, which describe 
the levels various features take up in creating a certain song. These included the following key parameters -

\section{Valence}

A measure from 0.0 to 1.0 describing the musical positiveness conveyed by a track. Tracks with high valence sound more positive (e.g., happy, cheerful, euphoric), while tracks with low valence sound more negative (e.g., sad, depressed, angry).

\section{Tempo}

The overall estimated tempo of a track in beats per minute (BPM). In musical terminology, tempo is the speed or pace of a given piece and derives directly from the average beat duration.

\section{Time Signature}

An estimated overall time signature of a track. The time signature (meter) is a notational convention to specify how many beats are in each bar (or measure).

\section{Speech}

Speech detects the presence of spoken words in a track. The more exclusively speech-like the recording (e.g., talk show, audio book, poetry), the closer to 1.0 the attribute value. Values above 0.66 describe tracks that are probably made entirely of spoken words. Values between 0.33 and 0.66 describe tracks that may contain both music and speech, either in sections or layered, including such cases as rap music. Values below 0.33 most likely represent music and other non-speech-like tracks.

\section{Mode}

Mode indicates the modality (major or minor) of a track, the type of scale from which its melodic content is derived. Major is represented by 1 and minor is 0 .

\section{Energy}

Energy is a measure from 0.0 to 1.0 and represents a perceptual measure of intensity and activity. Typically, energetic tracks feel fast, loud, and noisy. For example, death metal has high energy, while a Bach prelude scores low on the scale. Perceptual features contributing to this attribute include dynamic range, perceived loudness, timbre, onset rate, and general entropy.

\section{Instrumentalness}

Predicts whether a track contains no vocals. "Ooh" and "aah" sounds are treated as instrumental in this context. Rap or spoken word tracks are clearly "vocal". The closer the instrumentalness value is to 1.0, the greater likelihood the track contains no vocal content. Values above 0.5 are intended to represent instrumental tracks, but confidence is higher as the value approaches 1.0 .

\section{Key}

The key the track is in. Integers map to pitches using standard Pitch Class notation. E.g., $0=\mathrm{C}, 1=\mathrm{C} \sharp / \mathrm{Db}, 2$ $=\mathrm{D}$, and so on.

\section{Liveness}

Detects the presence of an audience in the recording. Higher liveness values represent an increased probability that the track was performed live. A value above 0.8 provides strong likelihood that the track is live.

\section{Loudness}

The overall loudness of a track in decibels (dB). Loudness values are averaged across the entire track and are useful for comparing relative loudness of tracks. Loudness is the quality of a sound that is the primary psychological correlate of physical strength 
(amplitude). Values typical range between -60 and 0 $\mathrm{db}$. We came to the conclusion that the optimal boundary conditions for filtering and selecting songs from the dataset would be "valence" and "energy".

This conclusion was reached as these two were the primary factors in deciding whether a song is perceived by a listener as a "positive" or a "negative" song. The songs which tend towards mean values in both the aforementioned parameters were classified as "neutral". The dataset was then linked and integrated with the application by importing it in the backend server. Pandas was then used to read and filter the dataset for the output on the website.

The main point emphasized here was this -

4.3.1 Any song with a valence greater than $0.4 \&$ an energy greater than 0.5 is considered POSITIVE.

4.3.2 Any song with a valence \& an energy tending towards the mean average values of the dataset is considered NEUTRAL.

4.3.3 Any song with a valence lesser than $0.4 \&$ an energy lesser than 0.5 is considered NEGATIVE

\section{TECHNOLOGIES AND TOOLS USED}

\section{Anaconda Distribution of Python 3.8}

With over 20 million users worldwide, the opensource Individual Edition (Distribution) is the easiest way to perform Python/R data science and machine learning on a single machine. Developed for solo practitioners, it is the toolkit that equips you to work with thousands of open-source packages and libraries.

2. Jupyter Notebook as IDE: The Jupyter Notebook is an open-source web application that allows you to create and share documents that contain live code, equations, visualizations and narrative text. Uses include: data cleaning and transformation, numerical simulation, statistical modeling, data visualization, machine learning, and much more.

3. Visual Studio Code: is a code editor that's redefined and optimized for building and debugging modern web and cloud applications.

4.SkLearn: Scikit-learn (formerly scikits. learn and also known as sklearn) is a free software machine learning library for the Python programming language.[3] It features various classification, regression and clustering algorithms including support vector machines, random forests, gradient boosting, $\mathrm{k}$-means and DBSCAN, and is designed to interoperate with the Python numerical and scientific libraries NumPy and SciPy.

5. Django 3.2: Django is a high-level Python Web framework that encourages rapid development and clean pragmatic design. A Web framework is a set of components that provide a standard way to develop websites fast and easily. Django's primary goal is to ease the creation of complex database-driven websites.

6. NLTK: is a leading platform for building Python programs to work with human language data. It provides easy-to-use interfaces to over 50 corpora and lexical resources such as WordNet, along with a suite of text processing libraries for classification, tokenization, stemming, tagging, parsing, and semantic reasoning, wrappers for industrial-strength NLP libraries, and an active discussion forum.
- Brown
- Webtext
- Lemmetizers
- Stopwords
- PortStemmer
- Tokenizer 
spaCy: is an open-source software library for advanced natural language processing, written in the programming languages Python and Cython. The library is published under the MIT license and its main developers are Matthew Honnibal and Ines Montani, the founders of the software company Explosion.

8. Pandas: is a Python package that provides fast, flexible, and expressive data structures designed to make working with structured (tabular, multidimensional, potentially heterogeneous) and time series data both easy and intuitive. It aims to be the fundamental high-level building block for doing practical, real world data analysis in Python.

9. TextBlob: is a Python (2 and 3) library for processing textual data. It provides a simple API for diving into common natural language processing (NLP) tasks such as part-of-speech tagging, noun phrase extraction, sentiment analysis, classification, translation, and more.

10.DjangoRESTFramework: (DRF) is a powerful and flexible toolkit for building Web APIs. Its main benefit is that it makes serialization much easier. Django REST framework is based on Django's classbased views and it adopts implementations such as class-based views, forms, model validator, Query Set, etc.

11. MonkeyLearn: is an AI platform that allows you analyze text with Machine Learning to automate business workflows and save hours of manual data processing.

12. Bootstrap: is an open-source toolkit for developing with HTML, CSS, and JavaScript
13.Visual Studio Code: is a code editor that's redefined and optimized for building and debugging modern web and cloud applications

\section{SALIENT FEATURE}

\section{[1] TO PROVIDE INTUITIVE MUSIC} RECOMMENDATION:

Our application has been built considering the foundations of existing music recommendation systems and their modus operandi. Existing systems rely heavily on user history and keyword searches, which could become obsolete with an advancement in technology. The platform we have created in this project is an initial attempt at providing music recommendations to the user purely based on an emotion that is deduced from their inputted text.

\section{[2] TO ENGAGE THE USER WITH NEW} MUSIC DISCOVERY:

Through the platform, not only do users come across music that accentuates or acts positively against the emotion they feel, but also brings up music suggestions that are previously unheard of for the user. It randomizes the choice of songs from a large dataset, providing new music with every request. This opens up the user to a world of new music, helps them discover new artists, and also refreshes and enhances their taste in music.

\section{FUNCTIONALITY}

Finding the correlation between the user data (e.g., location, time of the day, music listening history, emotion, etc.) and the music is challenging task.

Figure 1.1. Architecture of an Emotion-Aware Music Recommendation System 
The platform in our project provides simple functionalities to the user, with great ease of access and an aesthetic UI that complements the project -

- Sentiment Analysis with Emotion Deduction

- Curated Music Recommendation, according to the user's emotion

- An embedded music player, for instant access to music

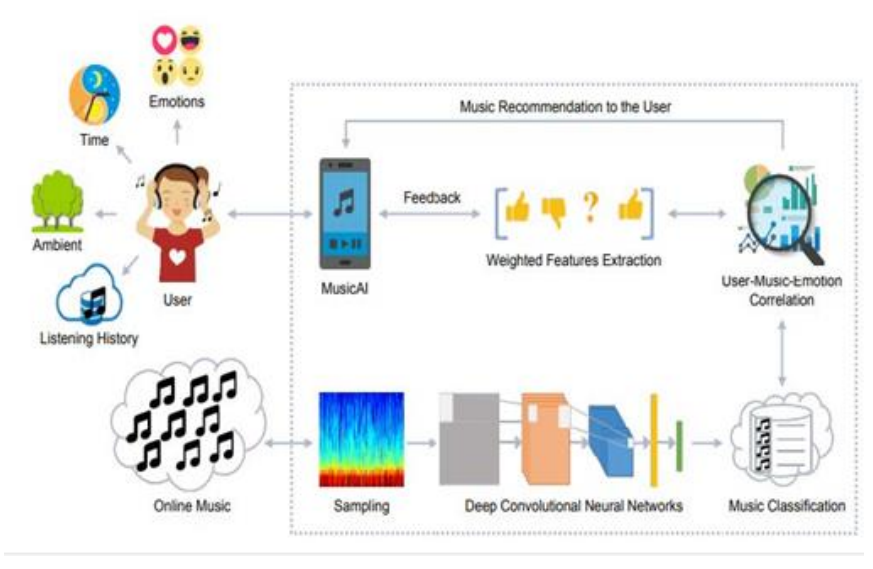

XI. IMPACT

Societal Impact

- Like addressed before, through our project we're aiming to provide accurate music recommendations for situations a human is in. Situations we're in have an impact on our emotional state and the way our mind functions. By giving accurate music we open/widen avenues of understanding these situations which in turn helps in understanding the state of a human which in its turn gives opportunity to address humane issues and provide comfort.

\section{Research Impact}

- The potential of data technology and machine learning in the online music world feels underused, and while research in various subtopics has been constructed, there is no sign yet of a complete amalgamation of these technologies for music.
- We seek to provide a platform which uses machine learning and data analysis in tandem with each other for this very purpose, which builds upon the user and its thoughts to provide a release of emotion.

\section{XII.ARCHITECTURE AND ENVIROMENT}

Our research revolves around understanding popular techniques of music recommendations. Our system encompasses a Web Server, Natural Language Processor, ML Model and Database of songs. Classifying different music based on their composition would require Signal Processing which is out of the scope of this project, hence readily available music dataset is used. Classification is done on the basis of use of instruments, tempo, time signature, genre etc. Natural Language Processor is used to understand scenarios given by the user. Output of this step is keywords with inferences. A Machine Learning Model is trained over the classified data. To map the Output of Natural of Language Processor with the ML Model for accurate recommendations protocols and rules need to be formulated. The block diagram shows how the raw classified music data is processed. The flowchart defines the steps that must be taken to get accurate music recommendations

\section{ACKNOWLEDGEMENT}

It is with a very warm sense of gratitude and privilege that we would like to acknowledge the efforts of the people who helped us with the ideation, creation and subsequent execution of this Final Year Project. Firstly, we would like to thank our Chief Project Mentor, Professor Sanjay Deshmukh, for pushing us beyond our limits in all the right directions with regards to our project. His omnipresent guidance and support have been invaluable to the delivery of our project. We would 
also like to thank our esteemed Dean, for handing us this opportunity to enhance and hone our skills in a practical application of our curriculum. We would also like to mention the constant support we have received from our classmates, if and when it was required. This project wouldn't have come such a long way without it. Lastly, our undying gratitude to the institution itself, for helping us develop our skills and build a foundation upon which this project could be laid in our final year as students.

\section{CONCLUSION AND RESULT}

Today, music is a very important and perhaps inseparable part of people's daily life. There are many genres of music and these genres are different from each other, resulting in people to have different preferences of music. As a result, it is an important and up-to-date issue to classify music and to recommend people new music in music listening applications and platforms. Most listening platforms offer music recommendation to their users by means of some methods such as meta-data analysis and collaborative filtering [18]

Our project aims at defining a mode for the recognition of a specific emotion within a text piece and the subsequent recommendation of music to the user, based on this extracted emotion. As mentioned earlier, the whole premise of our project was to redefine the use of data analysis and machine learning as one concept, when used in the spectrum of music. There lies untapped potential to this application itself, which can and will be looked into as time progresses, with the addition of a wider range of emotions, multiple emotions at a time, a much larger song database to choose from, and the fusion of user collaborative filtering to provide similar recommendations to a wider target audience based on the collected data history. The possibilities are endless, and with the successful implementation of this project, we find the motivation to drive this forward into the real world and make it available for the masses, sooner rather than later.

\section{FUTURE SCOPE}

Our platform has the potential to strive in an everchanging and improving world of technology and innovation, especially considering the fact that it proves to be beneficial or, at the least, supportive to a human cause at any given time. With heavier training, the model can be trained to target and study specific emotions instead of simplistic labels, thus providing an even more user-specific output based on the inputted text by the user. Considering the fact that the platform gives a list of songs as a music recommendation instantly when run, we feel it would work flawlessly in a voice- enabled assistant environment. Voice-enabled assistants are a modern marvel, and integrating THEO 's platform into voiceenabled assistants would allow users to speak directly into a device, with the device then deducing an emotion and immediately providing songs that act towards that emotion.

Lyrics-Based Recommendation: Let's say the sentence includes the word "anxious" then the song's lyrics that imply anxiety or has the word —anxious\| will be picked. Descriptive Recommendation: Let's say the sentence is "I was on a ship at night sad and lonely." As you can see this sentence is pretty descriptive, so the recommendations will be based on words like ship ', =night ', =lonely 'etc. Objective Recommendation: If the sentence contains the word guitar ', then the recommendations will also be based on it.

\section{INFERENCES}


The most perception made from the looked into papers are:

- FTAMR (Fine grained Time mindful music proposal), investigates the user's habit in a period and after that prescribes diverse tunes at diverse times.

- The K-nearest neighbor (K-NN) calculation will be utilized to tell music with the closest distance

- By clustering the client in progress, it can successfully decrease the calculation of the closeness between clients, and progress the running speed of the calculation. It is useful to move forward the exactness of the recommendation

- The labels not as it were serving as extra to broaden the substance of music but construct personalized music proposals for users.

\section{REFERENCES}

[1]. Mengxian. Li, Wenjun Jiang, Kenli. Li (2017). When and What Music Will You Listen to? : Fine-grained Time-aware Music Recommendation. 2017 IEEE International Symposium on Parallel and Distributed Processing with Applications and 2017 IEEE International Conference on Ubiquitous Computing and Communications (ISPA/IUCC). DOI: 10.1109/ISPA/IUCC.2017.00165

[2]. Hyojin Chin, Jayong Kim, Yoonjong Kim, Jinseop Shin, Mun. Y. Yi (2018). Explicit Content Detection in Music Lyrics Using Machine Learning.2018 IEEE International Conference on Big Data and Smart Computing. DOI: 10.1109/BigComp.2018.00085.

[3]. Jinhyuck Choi, Jin-Hee Song, Yanggon Kim (2018), An Analysis of Music Lyrics by Measuring the Distance of Emotion and Sentiment. 2018 19th IEEE/ACIS International Conference on Software Engineering, Artificial
Intelligence, Networking and Parallel/Distributed Computing (SNPD). DOI: 10.1109/SNPD.2018.8441085

[4]. Kunhui Lin*, Zhentuan Xu, Jie Liu, Qingfeng $\mathrm{Wu}$ and Yating Chen (2016), 2016 7th IEEE International Conference on Software Engineering and Service Science (ICSESS). DOI: 10.1109/ICSESS.2016.7883055

[5]. Kunhui Lin*, Zhentuan Xu, Jie Liu, Qingfeng $\mathrm{Wu}$ and Yating Chen (2016), 2016 7th IEEE International Conference on Software Engineering and Service Science (ICSESS) . DOI: 10.1109/ICSESS.2016.7883055

[6]. Mengsha Wang, Yingyuan Xiao, Wenguang Zheng, Ching-Hsien Hsu (2018). Tag-based Personalized Music Recommendation. 2018 15th International Symposium on Pervasive Systems, Algorithms and Networks. DOI: 10.1109/I-SPAN.2018.00040

[7]. A. Elbir and N. Aydin (2020), Music genre classification and music recommendation by using deep learning. Electronics Letters (Volume: 56, Issue: 12, 611 2020) DOI: 10.1049/el.2019.4202

[8]. Nida Manzoor Hakak, Mohsin Mohd, Mahira Kirmani Mudasir mohd 2017 International Conference on Computer, Communications and Electronics (Comptelix) DOI: 10.1109/COMPTELIX.2017.8004002

[9]. Khodijah Hulliyah, Normi Sham Awang Abu Bakar, Amelia Ritahani Ismail 2017 Second International Conference on Informatics and Computing (ICIC) DOI: 10.1109/IAC.2017.8280568

[10]. Lutfun Nahar, Chittagong, Zinnia Sultana 1st International Conference on Advances in Science, Engineering and Robotics Technology 2019 (ICASERT 2019) DOI: 10.1109/ICASERT.2019.8934654

[11]. Samar Al-Saqqa, Heba Abdel-Nabi, Arafat Awajan 2018 8th International Conference on 
Computer Science and Information Technology (CSIT) DOI: 10.1109/CSIT.2018.8486405

[12]. Xinzhi Wang,Luyao Kou,Vijayan Sugumaran,Xiangfeng Luo,Hui Zhang IEEE Transactions on Cybernetics ( IF 11.079 ) Pub Date : 2020-05-12 DOI: 10.1109/tcyb.2020.2987064

[13]. Ashok Kumar, J L Mazher Iqbal International Journal of Engineering and Advanced Technology (IJEAT) ISSN: 2249 - 8958, Volume-9 Issue-1S5, December, 2019 DOI: 10.35940/ijeat. A1068

[14]. Jose Camacho-Collados, Mohammad Taher Pilehvar Kim et al., 2016; Xiao and Cho, 2016) or word senses (Li and Jurafsky, 2015; Flekova and Gurevych, 2016; Pilehvar et al., 2017)

[15]. Jongpil Lee, Jiyoung Park Keunhyoung Luke Kim Juhan Nam c 2017 Jongpil Lee et al. This is an open-access article distributed under the terms of the Creative Commons Attribution 3.0 Unported License.

[16]. Douglas Eck, Thierry Bertin-Mahieux, Paul Lamere IEEE Transactions on Cybernetics ( IF 11.079 ) Pub Date : 2019-03-

[17]. Ashu Abdul ,Jenhui Chen ,Hua-Yuan Liao ,Shun-Hao Chang Appl. Sci. 2018, 8(7), 1103; https://doi.org/10.3390/app8071103 Received: 31 May 2018 / Revised: 25 June 2018 / Accepted: 6 July 2018 / Published: 8 July 2018

[18]. Soujanya Poria, Iti Chaturvedi, Erik Cambria, Amir Hussain 2016 IEEE 16th International Conference on Data Mining 2374-8486/16 \$31.00 @ 2016 IEEE DOI 10.1109/ICDM.2016.1

\section{Cite this article as :}

Vipin Wani, Niketan Bothe, Avani Soni, "Music Suggestion Via Sentimental Analysis of User-Inputted Texts", International Journal of Scientific Research in Computer Science, Engineering and Information Technology (IJSRCSEIT), ISSN : 2456-3307, Volume 7 Issue 3, pp. 51-66, May-June 2021. Available at doi : https://doi.org/10.32628/CSEIT217320 Journal URL : https://ijsrcseit.com/CSEIT217320 